Research paper

\title{
Exploiting the impact of the secretome of MSCs isolated from different tissue sources on neuronal differentiation and axonal growth
}

\author{
Rita Catarina Assunção-Silva a, b, c, Bárbara Mendes-Pinheiro ${ }^{\text {a, b }}$, Patrícia Patrício a, b, c, \\ Leo A. Behie ${ }^{d}$, Fábio Gabriel Teixeira ${ }^{\text {a, b }}$, Luísa Pinto a, b, c , António José Salgado a, b, * ' \\ a Life and Health Sciences Research Institute (ICVS), School of Medicine, University of Minho, Campus de Gualtar, 4701-057, Braga, Portugal \\ ${ }^{\mathrm{b}}$ ICVS/3B's - PT Government Associate Laboratory, Braga, Guimarães, Portugal \\ ${ }^{c}$ BnML, Behavioral and Molecular Lab, Braga, Portugal \\ ${ }^{\mathrm{d}}$ Pharmaceutical Production Research Facility, Schulich School of Engineering, University of Calgary, 2500 University Drive NW, Calgary, Alberta, Canada
}

\section{A R T I C L E I N F O}

\section{Article history:}

Received 14 February 2018

Accepted 31 July 2018

Available online xxx

\section{Keywords:}

Mesenchymal stem cells

Secretome

Neuroregulatory factors

Neurodifferentiation

Axonal outgrowth

Cell-free based CNS therapy

\begin{abstract}
A B S T R A C T
Cell transplantation using Mesenchymal stem cell (MSC) secretome have recently been presented as a possible free-based therapy for CNS related disorders. MSC secretome is rich in several bio-factors that act synergically towards the repair of damaged tissues, thus making it an ideal candidate for regenerative applications. Great effort is currently being made to map the molecules that compose the MSC secretome. Previous proteomic characterization of the secretome (in the form of conditioned media - CM) of MSCs derived from adipose tissue (ASC), bone-marrow (BMSC) and umbilical cord (HUCPVC) was performed by our group, where proteins relevant for neuroprotection, neurogenic, neurodifferentiation, axon guidance and growth functions were identified. Moreover, we have found significant differences among the expression of several molecules, which may indicate that their therapeutic outcome might be distinct. Having this in mind, in the present study, the neuroregulatory potential of ASC, BMSC and HUCPVC CM in promoting neurodifferentiation and axonal outgrowth was tested in vitro, using human telencephalon neuroprogenitor cells and dorsal root ganglion explants, respectively. The CM from the three MSC populations induced neuronal differentiation from human neural progenitor cells, as well as neurite outgrowth from dorsal root ganglion explants. Moreover, all the MSC populations promoted the same extent of neurodifferentiation, while ASC CM demonstrated higher potential in promoting axonal growth.
\end{abstract}

(C) 2018 Published by Elsevier B.V.

\section{Introduction}

An extensive body of literature suggests that Mesenchymal stem cell (MSC)-mediated paracrine activity plays a role in promoting tissue repair. MSCs were firstly identified by Friedenstein as multipotent stem cells characterized by the capacity to self-renew, to adhere to plastic and colonize, and to differentiate into three mesodermal cell lineages [1]. However, the real interest around MSCs is their contribution towards regeneration of tissues upon

\footnotetext{
* Corresponding author. Life and Health Sciences Research Institute (ICVS), School of Medicine, University of Minho, Campus de Gualtar, 4701-057, Braga, Portugal.

E-mail addresses: id5818@alunos.uminho.pt (R.C. Assunção-Silva), id7153@ alunos.uminho.pt (B. Mendes-Pinheiro), patriciapatricio@med.uminho.pt (P. Patrício), b6986@med.uminho.pt (F.G. Teixeira), luisapinto@med.uminho.pt (L. Pinto), asalgado@med.uminho.pt (A.J. Salgado).
}

injury. In fact MSC-based therapies have been used in the context of several neurodegenerative diseases, where neuronal survival has been reported in animal models of stroke [2] and traumatic brain injury (TBI) [3]. Additionally, the recovery of motor function of induced models of Parkinson's disease (PD) [4] and SCI, with an observed remyelination and reconnection of the neural circuitry [5,6], has also been observed upon MSCs administration. Direct evidences attribute the regenerative potential of MSCs to their ability to secrete several biomolecules and trophic factors, namely neurotrophic growth factors, chemokines, cytokines, and extracellular matrix proteins, as well as extracellular vesicles, that might be relevant in a clinical setting [7-10]. In the context of neuroregeneration, pre-clinical and clinical findings show that these molecules can directly stimulate the recruitment, proliferation and differentiation of the endogenous cells [11,12]. Additionally, they can regulate local mechanisms such as apoptosis, scarring and revascularization, as well as modulate immune and inflammatory 
responses [9,13], thus contributing to reduce tissue damage.

Recent insights on the effective therapeutic role of MSCsecreted bio-factors, especially given the fact that MSCs have a limited engraftment and survival rate when delivered into a damaged tissue [14], opens the possibility of using MSC secretome as a cell-transplantion free based regenerative therapy. In fact, the therapeutic application of the secreted molecules in replacement of stem cells presents enormous advantages as it should minimize stem cell-related ethical and immune-compatibility issues [15], as well as allow a precise dosing and localized delivery to the damaged tissues in a minimally invasive manner [16].

The perspective of safer and more effective strategies motivated further investigation towards the identification of the molecules composing the cell secretome, rather than only looking to its overall regenerative effects. In this line, proteomic profiling of MSC conditioned media $(\mathrm{CM})$ became intensively explored in recent years [17-21].

While valuable tools for MSC secretome characterization were being used, several studies highlighted significant differences between different tissue-sources of MSCs. In fact, the heterogeneity of MSCs residing in different tissues has been reported some time ago [10]. So far, the best characterized and the most studied sources of adult MSCs are obtained from the bone-marrow (BMSCs), adipose tissue (ASCs) and umbilical cord. Several studies provided us with distinct characteristics of each population, that goes from different expression of cell surface markers [22], to specific differentiation processes [23] and immunomodulatory functions [24]. A relevant point of interest that arises from a collective analysis on this heterogeneity studies is the fact that different tissue sources of MSCs are likely to have different secretion profiles [19,25]. For instance, we have previously shown that the exposure of primary cultures of hippocampal neurons to the CM of ASCs and Human Umbilical Cord Perivascular Cells (HUCPVCs) had different effects on cell proliferation and metabolic activity [26]. Hsieh and colleagues also found that MSCs isolated from Wharton's jelly secreted more factors related to angiogenesis and neurogenesis than BMSCs, which improved neural differentiation and migration and decreased cell apoptosis in an in vitro model of acute ischemic stroke [27]. Considering the existence of such differences on the secretome composition of MSCs obtained from different tissue-sources, the choice of the best MSC population for a particular application must be determined according to their characteristics and secretory profile. Thereafter, our group found that it is crucial to perform a detailed mapping of the CM obtained from BMSCs, ASCs and HUCPVCs. In a recently published proteomic analysis, we have shown that all these populations were able to secret important factors known to be involved in processes of several CNS disorders/ injuries [28]. Moreover, the pattern and composition of ASCs, BMSCs and HUCPVCs CM differed, a fact that could indicate a certain degree of specificity towards different CNS related conditions. After this, it remains unclear if these different secretion profiles could activate distinct mechanisms by which the repair and regeneration of tissues from the nervous system may be regulated. Based on these results, the present study proposes to further explore the previously analyzed MSC CM potential in promoting the differentiation and axonal growth of neural populations in vitro.

\section{Materials and methods}

\subsection{Cell culture}

2.1.1. Human bone marrow mesenchymal stem cells (BMSCs), adipose tissue derived stem cells (ASCs), and human umbilical cord perivascular cells (HUCPVCS)

BMSCs (Stem Cell Technologies, Grenoble France) were thaw and expanded according to protocol established in our lab [29]; ASCs were kindly provided by Professor Gimble (Pennington Biomedical Research Center/Tulane University, USA); and HUCPVCs were kindly provided by Professor Davies (University of Toronto, Canada). ASCs and HUCPVCs were isolated as previously described [30,31].

Cells were cultured and maintained in $\alpha$-MEM medium (Invitrogen, USA) supplemented with sodium bicarbonate $\left(\mathrm{NaHCO}_{3}\right.$; Merck, USA), 10\% of fetal bovine serum (FBS; Biochrom, Germany) and $1 \%$ Penicilin-Streptomycin antibiotic (P/S; Invitrogen, USA). Upon confluence, cells were enzymatically dissociated with $0.05 \%$ trypsin/EDTA (Invitrogen, USA), re-plated at a density of 4000 cells/ $\mathrm{cm}^{2}$ and maintained at $37^{\circ} \mathrm{C}, 5 \%$ humidified $\mathrm{CO}_{2}, 95 \%$ air and $90 \%$ relative humidity.

\subsubsection{Human telencephalon Neural Progenitor Cells (htNPCS)}

htNPCs were isolated from 10-week human fetus telencephalon region, as previously described [31]. Ethical consent was approved by the Conjoint Health Research Ethics Board (CHREB), University of Calgary (ID: E-18786). htNPCs were thaw and plated in Nunc T25 fask containing $5 \mathrm{~mL}$ of a serum-free medium PPRF-h2, described in detail by Baghbaderani et al. [32]. Cells were maintained in culture for $48 \mathrm{~h}$, during which aggregated into neurospheres. After this time, htNPCs were mechanically dissociated and re-plated into fresh medium. Every 4 days, $40 \%$ of the medium was replaced by fresh.

\subsection{Conditioned media (CM) collection}

The CM of ASCs, HUCPVCs and BMSCs was collected from cells in passage 5 . For that, cells were plated at a density of 4000 cells $/ \mathrm{cm}^{2}$, and allowed to grow for $72 \mathrm{~h}$. After this, cells were washed 5 times with PBS without $\mathrm{Ca}^{2+}$ and $\mathrm{Mg}^{2+}$ (Invitrogen, USA), and once with the conditioning medium.

For neurodifferentiation experiments, Neurobasal A medium (Invitrogen, USA) supplemented with 1\% Kanamycin (Invitrogen, USA) was added to the cells. For axonal growth assays, Neurobasal medium (Invitrogen, USA) supplemented with $1 \%$ Pen-Strep was used. After $24 \mathrm{~h}$ of conditioning period, the $\mathrm{CM}$ was collected and frozen at $-80^{\circ} \mathrm{C}$ until used.

\section{3. htNPCs culture with MSC CM}

For neurodifferentiation experiments, htNPCs were enzymatically dissociated with $0.05 \%$ trypsin-EDTA, and plated onto glass coverslips pre-coated with poly-D-lysine hydrobromide $(100 \mu \mathrm{g} /$ $\mathrm{mL}$; Sigma) and laminin $(10 \mu \mathrm{g} / \mathrm{mL}$; Sigma $)$ at a density of $5.5 \times 104$ cells. Cells were maintained in culture for 5 days with the $\mathrm{CM}$ collected from the three MSC populations, at $37{ }^{\circ} \mathrm{C}, 5 \% \mathrm{CO} 2,95 \%$ air and $90 \%$ relative humidity. htNPCs culture with Neurobasal-A medium supplemented with $1 \%$ of kanamycin was used as control.

\subsection{Isolation and culture of dorsal root ganglion (DRG) explants with MSC CM}

Dorsal root ganglion explants were used for axonal growth experiments. For that, DRGs from 5 days-old neonatal Wistar-Han rat pups were dissected as previously described [33]. Briefly, DRGs from cervical and thoracic regions of the spine of neonatal rat pups (P5) were dissected and the remnants of peripheral nerve processes were cleaned. The explants were placed on top of collagen hydrogels, prepared as previously described [34] and incubated with MSC CM for 7 days. DRG cultures in collagen gels in Neurobasal Medium supplemented with B27, L-glutamine, glucose and $1 \%$ of $\mathrm{P} / \mathrm{S}$ was used as control. 


\subsection{Immunostaining}

htNPCs. htNPCs were fixed with, washed, and blocked as previously described [4], with the following primary antibodies: rabbit anti-doublecortin (DCX; 1:500, Abcam, Cambridge, MA, USA) to detect immature neurons, and mouse anti-rat microtubule associated protein-2 (MAP-2; 1:500, Sigma), for $1 \mathrm{~h}$ at $37^{\circ} \mathrm{C}$. After washing, samples were incubated with the secondary antibodies Alexa Fluor 488 goat anti-rabbit immunoglobulin G (IgG, Life Technologies) and Alexa Fluor 594 goat anti-mouse immunoglobulin G (IgG, Life Technologies) for $1 \mathrm{~h}$ at $37^{\circ} \mathrm{C}$. Further incubation with 4-6-diamidino-2-ph enylindole-dihydrochloride (DAPI; Life Technologies) was performed for $10 \mathrm{~min}$ at Room Temperature (RT). Samples were then observed under an Olympus BX-61 Fluorescence Microscope (Olympus, Hamburg, Germany).

DRGs. For the immunocytochemistry (ICC) of DRGs, the following antibodies were used: Mouse monoclonal anti-human neurofilament $200 \mathrm{kDa}$ (Millipore) as the primary antibody and Alexa fluor 488 goat anti-mouse IgG (Invitrogen) as the secondary antibody. DRGs were fixed with $4 \%$ paraformaldehyde (PFA) in PBS for $45 \mathrm{~min}$ at RT and washed with PBS. A further incubation $0.3 \%$ Triton X-100 (Sigma, USA) for 10 min at RT was used for cell permeabilization, and washing with PBS. Samples were then incubated with a blocking buffer solution [PBS containing 10\% fetal bovine serum (FBS)] for $90 \mathrm{~min}$ at RT, after which they were incubated with the primary antibody (diluted 1:200 in PBS solution with 10\% FBS) for $48 \mathrm{~h}$ at $4{ }^{\circ} \mathrm{C}$. After washed with a PBS solution containing $0.5 \%$ FBS, samples were incubated with the secondary antibody (diluted $1: 1000$ in PBS/0.5\%FBS solution) overnight at $4{ }^{\circ} \mathrm{C}$. After PBS washing, DAPI ( $1 \mu \mathrm{g} \mathrm{mL}-1$; Invitrogen) was added to the samples for $10 \mathrm{~min}$ to stain cell nuclei. Samples were finally washed and maintained hydrated in PBS for fluorescence microscopy analysis (Olympus BX-61 Fluorescence Microscope, Olympus, Hamburg, Germany).

\subsection{Neurodifferentiation assessment}

Neurodifferentiation of htNPCs was determined by qRT-PCR for NeuroD1 (ND1) and BIII-Tubulin (BIII-Tub) (details bellow), and by the number of Doublecortin (DCX) and Microtubule associated protein (MAP-2) expressing cells. For this purpose, three coverslips and ten representative fields per condition were chosen and imaged using a fluorescence microscope as referred above. To normalize the data between the different experiments, the results are presented in percentage (\%) of cells. This was calculated by counting the number of cells with positive staining for DCX and
MAP-2 markers, and dividing this value by the total number of cells/field (DAPI-positive cells; $\mathrm{n}=3$ ).

\subsection{Protein association network analysis and quantitative real time $P C R$}

For neurodifferentiation experiments, the mRNA expression levels of selected genes of interest (Table 1) were measured by quantitative real time polymerase chain reaction (qRT-PCR), after htNPCs incubation with MSC CM. These genes were selected based on protein-protein interaction networks from the previously identified biomolecules in the MSC CM [28] using STRING (Search Tool for the Retrieval of Interacting Genes/Proteins) bioinformatics tool. The neurodifferentiation markers NeuroD1 and Tubulin beta 3 Class III (TUBB3) were also used to assess the neurodifferentiation stage after incubation of htNPCs with MSC CM. The oligonucleotide primers for the target genes were designed using Primer-BLAST software (NCBI). The real time reactions were performed in an Applied Biosystems 7500 Fast Real-Time PCR System (Applied Biosystems, LLC, CA, USA) using PerfeCTa SYBR Green SuperMix, Low ROX (Quanta Biosciences). Target gene expression levels were normalized against the housekeeping gene Beta2-microglobulin (B2M), and presented as fold-change of mRNA levels compared to the Control group. The $2^{-\Delta \Delta C T}$ method was used to calculate foldchange levels.

\subsection{Axonal outgrowth/migration quantification}

As previously described [34], DRG axonal growth was inferred by the quantification of the area occupied by the neurites within the collagen gels. For that, after confocal imaging, samples $(n=8 /$ condition) were analyzed by Image J software. The image scale was first set and converted to 8 bit and binary, after which the body of the DRG itself was excluded. Thereafter, the area occupied by the neurites were automatically calculated considering the image black background as contrast. The area of neurite outgrowth is presented as $\mu \mathrm{m}^{2}$.

\subsection{Statistical analysis}

Statistical evaluation was performed using GraphPad Prism (version 5.0; GraphPad Software, USA). Differences among groups were assessed using Student's t-test or One-way ANOVA test. A pvalue of $\leq 0.05$ (95\% confidence level) was set as the criteria for statistical significance $\left.{ }^{*}\right)$.

Table 1

Forward and reverse sequences of oligonucleotide primers used in the qRT-PCR, and the respective gene symbol, name and product size.

\begin{tabular}{|c|c|c|c|}
\hline Gene symbol & Gene name & Primer sequence $5^{\prime}-3^{\prime}$ forward $=$ reverse & Product Size (bp) \\
\hline TUBB3 & Tubulin beta 3 class III & $\begin{array}{l}\text { Fw GGC CTC TTC TCA CAA GTA CG } \\
\text { Rv CCA CTC TGA CCA AAG ATG AAA }\end{array}$ & 317 \\
\hline NeuroD1 & Neuronal differentiation 1 & $\begin{array}{l}\text { Fw CCG TCC GCC GAG TTT G } \\
\text { Rv GCG GTG CCT GAG AAG ATT G }\end{array}$ & 173 \\
\hline FLT1 & Fms-related tyrosine kinase- 1 & $\begin{array}{l}\text { Fw CTG GGC AGC AGA CAA ATC CT } \\
\text { Rv AAA AGT CAC ACC TTG CTT CGG }\end{array}$ & 113 \\
\hline NRP1 & Neuropilin 1 & $\begin{array}{l}\text { Fw CGC AAG GCG AAG TCT TTT GA } \\
\text { Rv TGT GAG CTG GAA GTC ATC ACC }\end{array}$ & 265 \\
\hline TGFBR1 & Transforming growth factor beta receptor 1 & $\begin{array}{l}\text { Fw TCC AAC TAC TGG TTT ACC ATT GC } \\
\text { Rv TTC TTC TCC CCG CCA CTT TC }\end{array}$ & 123 \\
\hline SMAD2 & SMAD family member 2 & $\begin{array}{l}\text { Fw GGC CTT TAC AGC TTC TCT GAA CA } \\
\text { Rv ACTGGAGGCAAAACTGGTGTC }\end{array}$ & 240 \\
\hline STAT3 & Signal transducer and activator of transcription 3 & $\begin{array}{l}\text { Fw GGA GAA GGA CAT CAG CGG TAA } \\
\text { Rv GCT CTC TGG CCG ACA ATA CT }\end{array}$ & 205 \\
\hline B2M & Beta2-microglobulin (Reference gene) & $\begin{array}{l}\text { Fw GAT AGT TAA GTG GGA TCG AG } \\
\text { Rv GCA AGC AAG CAG AAT TTG GA }\end{array}$ & 95 \\
\hline
\end{tabular}


A

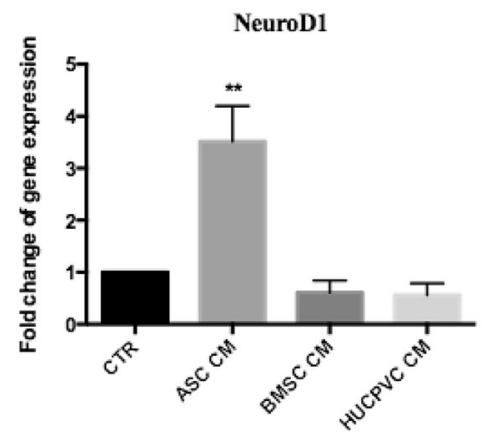

B

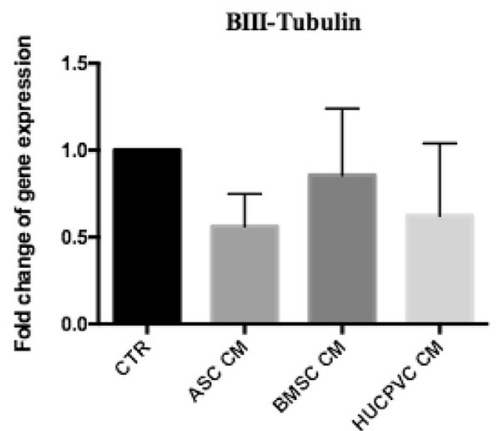

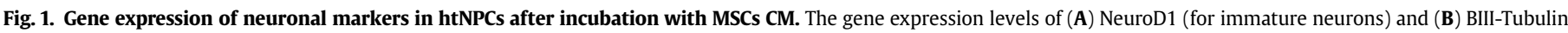

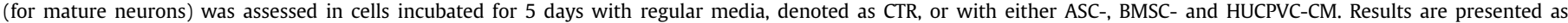
Mean $\pm \mathrm{SD} ; \mathrm{n}=3$ per condition; ${ }^{* *} \mathrm{p}<0.01$.

\section{Results and discussion}

\subsection{MSC CM induced htNPCs neuronal differentiation}

To assess the potential of MSC secretome in promoting neuronal differentiation, htNPCs were cultured with ASC, BMSC and HUCPVC $\mathrm{CM}$. After 5 days in culture, gene expression analysis of NeuroD1, a neuronal cell-fate and early differentiation marker, and Tubulin beta III (BIII-Tubulin), a marker of mature neurons, was performed in htNPCs to assess the differentiation stage of these cells (Fig. 1).

While NeuroD1 transcriptional expression was particularly overexpressed in htNPCs incubated with ASC CM (Fig. 1A), BIIItubulin was similarly expressed in all the experimental groups, with no statistically significant differences comparing with the control (Fig. 1B).

The differentiation of htNPCs was further confirmed by immunocytochemistry analysis for DCX and MAP-2, staining for immature- and early stage mature neurons, respectively. Higher levels of
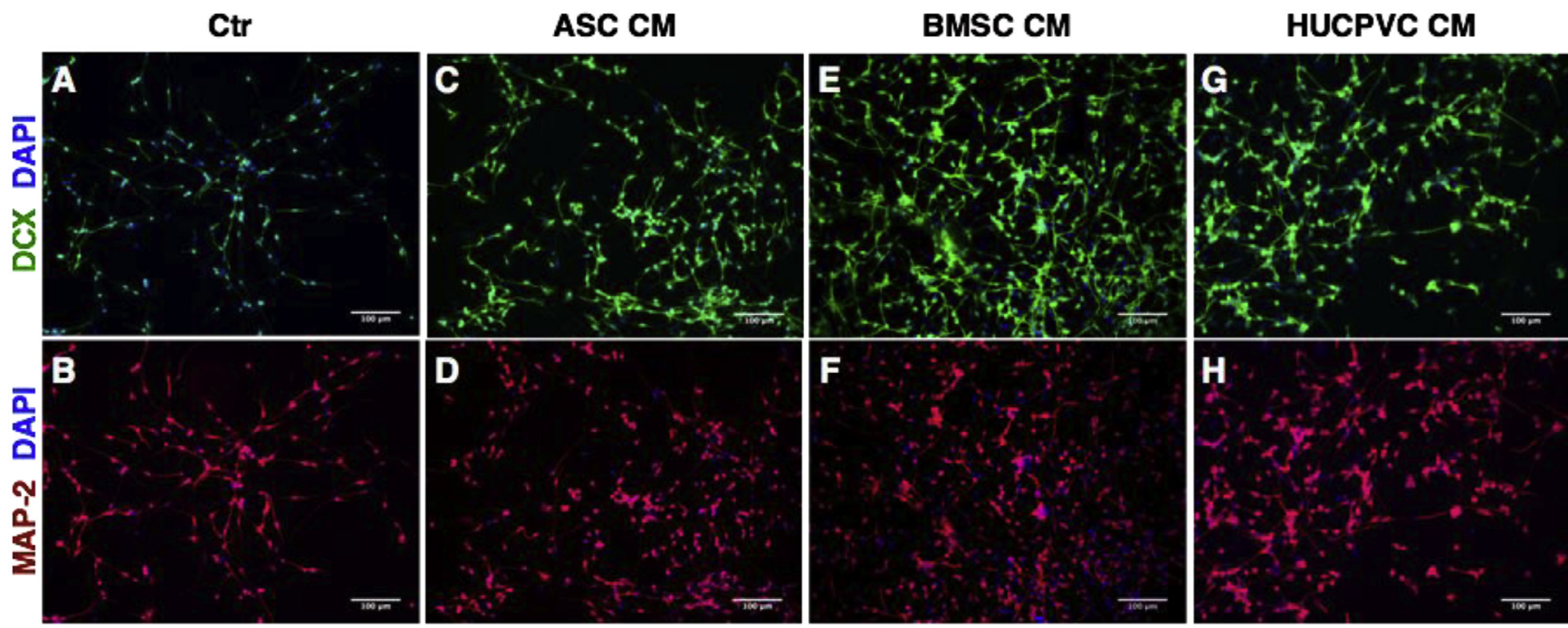

I

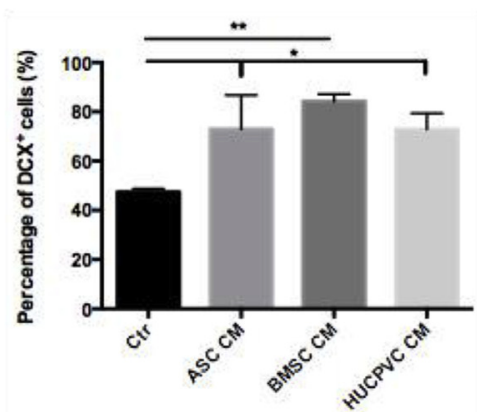

J

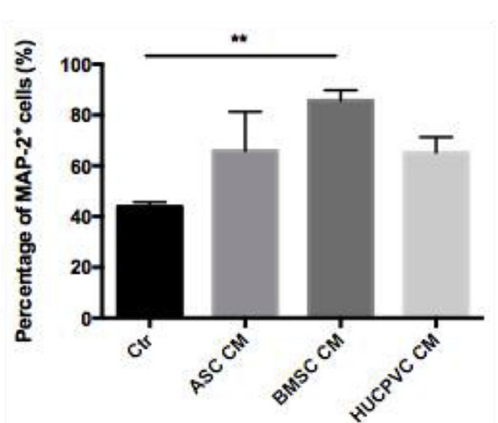

$\mathbf{K}$

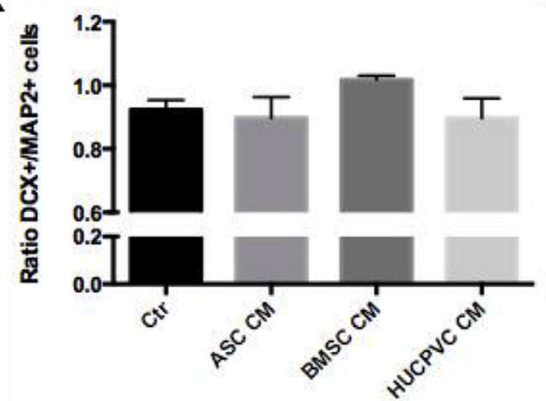

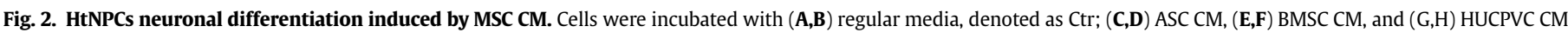

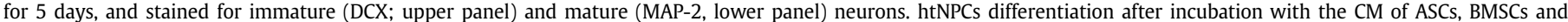

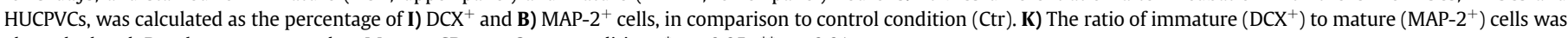
also calculated. Results are presented as Mean $\pm \mathrm{SD} ; \mathrm{n}=3$ per condition; ${ }^{*} \mathrm{p}<0.05 ;{ }^{* *} \mathrm{p}<0.01$. 

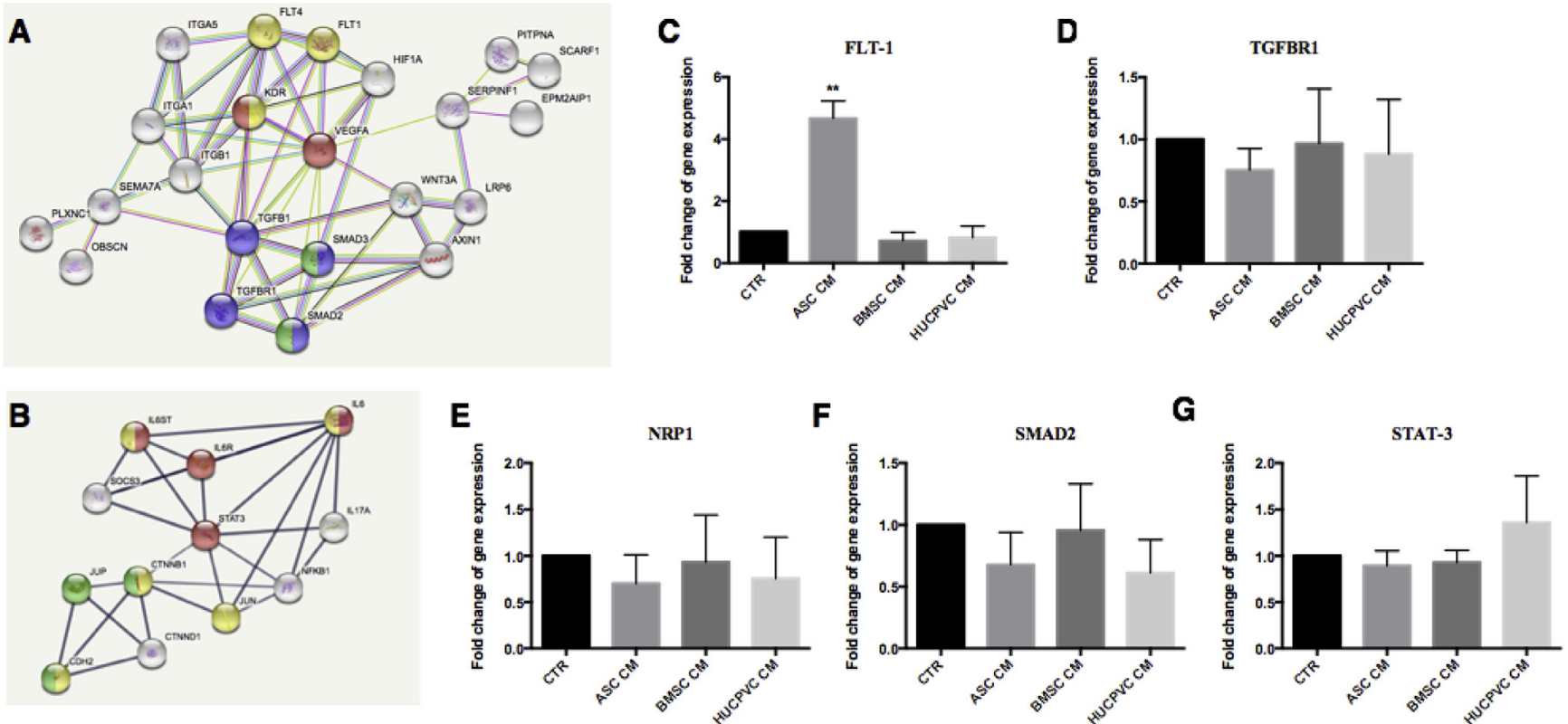

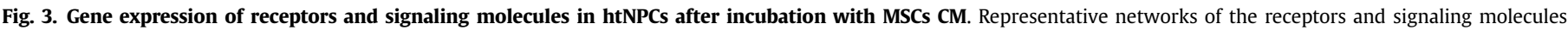

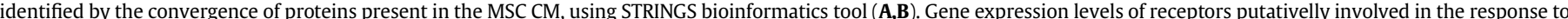

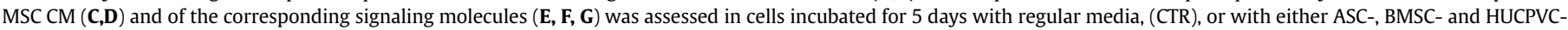
$\mathrm{CM}$. Results are presented as Mean $\pm \mathrm{SD} ; \mathrm{n}=3$ per condition; ${ }^{* *} \mathrm{p}<0.01$.

differentiation into a neuronal phenotype were revealed for all the $\mathrm{CM}$ (Fig. 2C-H). Indeed, as shown in Fig. 2 the CM of all MSC populations was able to induce neural differentiation of human fetal htNPCs (ASCs: $72,98 \pm 13,75 \%$; BMSCs: $84,20 \pm 2913 \%$; and HUCPVCs: $72,88 \pm 6490 \%$ ), with significant differences being obtained towards control samples (Ctr: 47,49 $\pm 2208 \%$ ).

Similar results were obtained for the differentiation of htNPCs into mature neurons (MAP-2 ${ }^{+}$cells; Fig. 2J). All MSC CM induced neuronal cell differentiation (ASCs: 65,88 $\pm 15,38 \%$; BMSCs: $85,67 \pm 4088 \%$; HUCPVCs: $65,24 \pm 6063 \%$ ), with no significant differences between them. Moreover, only BMSC CM was found to significantly increase the percentage of MAP- $2^{+}$cells in comparison to control (Ctr: $43,84 \pm 3240 \%$ ). Finally, the ratio of immature $\left(\mathrm{DCX}^{+}\right)$to mature (MAP-2 ${ }^{+}$) cells was found to be similar between all the experimental groups (Fig. $2 \mathrm{~K}$ ).

To investigate whether the CM effects on htNPCs neurodifferentiation may result from the presence of proteins that have been previously identified in the CM of these MSC populations, namely the Pigment epithelium-derived factor (PEDF, also known as SERPIN1), Semaphorin 7A (SEM7A), Cadherin2 (CHD2) and Interleukin-6 (IL-6) [28], we analyzed the gene expression for some of these factors' receptors in htNPCs after incubation with CM (Fig. 3). In order to identify converging receptors and signaling molecules that could be involved in the molecular response to these proteins we used STRINGs bioinformatics tool, and identified two protein-protein interaction-based networks, one that includes SERPIN1 and SEM7A (Fig. 3A), and another including CDH2 and IL-6 (Fig. 3B). In the first network, VEGF and TGFB were identified as common effector molecules. Thus, the gene expression of FLT-1 (Fig. 3D) and TGFBR1 (Fig. 3E), the receptors for VEGF and TGFB, respectively, was assessed as well as the related-signaling molecules NRP1 (Fig. 3F) and SMAD2 (Fig. 3G). The signaling molecule STAT3 was also assessed as it responds to both IL-6 and CDH2.

Gene expression results show that cells from all experimental groups express the receptors necessary to respond to the factors present in the MSC CM. The expression of FLT- 1 was significantly increased in htNPCs after incubation with ASC CM, when compared to Ctr, BM and HUCPVC CM. However, no variations were found in the expression of TGFBR1, NRP1, SMAD2, and STAT3 between the experimental groups.

\subsection{Axonal growth promoted by MSC CM}

The MSC CM-mediated axonal growth was herein studied using a DRG-based neurite outgrowth in vitro model. DRG explants were placed on top of collagen matrices, and the CM collected from ASCs, BMSCs and HUCPVCs were added to the culture for 7 days. The neurites grown from the explants were stained for Neurofilament and analyzed by fluorescence microscopy, as represented in Fig. 4.
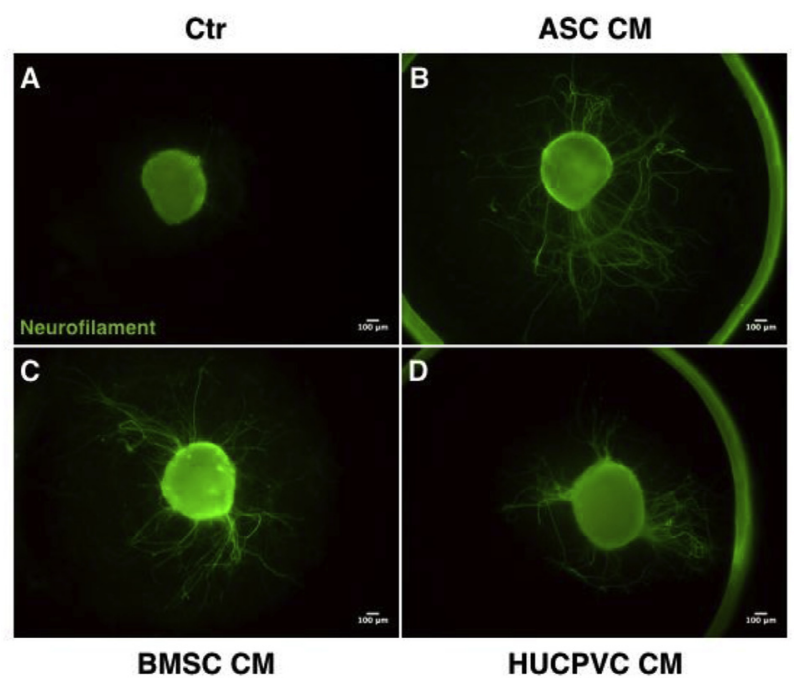

Fig. 4. Neurite outgrowth from DRG explants incubated with MSC CM. DRG staining against neurofilament was performed after culture with CM collected from B) ASCs (ASC CM), C) BMSCs (BMSC CM) and D) HUCPVCs (HUCPVC CM). Control conditions (A), denoted as Ctr, regards to DRGs incubated with supplemented Neurobasal medium (please see M\&M section). Scale bar: $100 \mu \mathrm{m}$. 
DRGs were found to be able to extend long neurites in the presence of ASC (Fig. 4A), BMSC (Fig. 4C) and HUCPVC CM (Fig. 4D). The same was not found for the control condition (Ctr; Fig. 4A), as almost no neurite growth was observed.

This qualitative analysis was confirmed by quantifying the area of neurite outgrowth (denoted as $\mu \mathrm{m}^{2}$ ) in the explants for each referred condition (Fig. 5).

Axonal growth was significantly increased in the presence of ASC CM $\left(520952 \pm 228401 \mu \mathrm{m}^{2}\right)$, when compared to control (Ctr; $\left.5589 \pm 9979 \mu \mathrm{m}^{2}\right)$. In addition, ASC CM induced significantly higher axonal growth than BMSC and HUCPVC CM $\left(194926 \pm 139336 \mu \mathrm{m}^{2}\right.$; $196718 \pm 129509 \mu \mathrm{m}^{2}$, respectively). This suggests that the ASC secretome provides a more robust and adequate environment for DRG axons to grow and migrate.

\section{Discussion}

Mapping the molecules that compose the secretome of MSCs aims at determining the factors upregulated by these cells in response to specific triggers, and thereafter finding their role in mediating one or more mechanisms of repair. Until recently, most reports focused on identifying only a subset of factors released by MSCs at high levels, therefore providing only a very superficial knowledge on the composition of its secretome. Contrary to this, a comparative analysis of the proteins secreted by ASCs, BMSCs and HUCPVCs was recently performed by our group using a more integrated proteomic approach, named Liquid chromatography tandem-mass spectrometry (LC-MS/MS) [28]. In that study, a vast panel of proteins with neuroprotection, neurogenic, neurodifferentiation, and axon guidance and growth functions was identified. The three MSC populations differed in their secretion profile, posing the question of whether their neuroregulatory action may differ accordingly. Therefore, the study herein presented aimed at evaluating the role of MSC secretome in mediating neurodifferentiation and axonal growth as a function of tissue source. For that purpose, htNPCs and DRGs were incubated with the CM of ASCs, BMSCs and HUCPVCs, for neurodifferentiation and axonal growth assessment, respectively.

htNPCs are normally expanded as neurospheres in the presence of a serum-free medium PPRF-h2, as demonstrated by Teixeira et al. [4]. Upon removal of the expansion medium, these cells are described to spontaneously differentiate into neural phenotypes. For that reason, this cell population was used in this study to test the neurodifferentiation potential of MSC CM. The incubation of htNPCs with the MSC CM induced significantly higher levels of differentiation into neuronal phenotypes in comparison to control conditions, as shown by the expression of DCX (immature neurons)

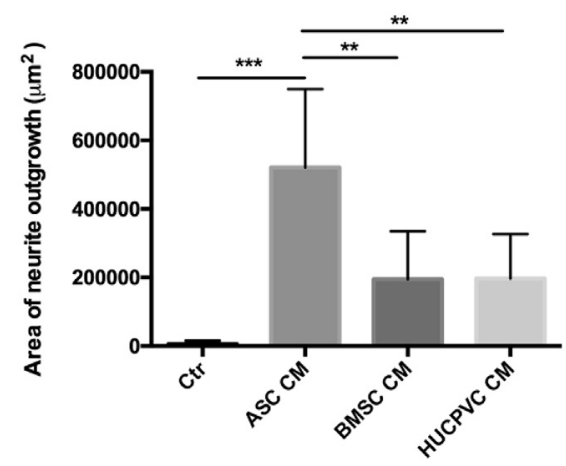

Fig. 5. Area of neurite outgrowth promoted by ASC, BMSC and HUCPVC CM, in comparison to control (Ctr). The mean area occupied by neurites $\left(\mu \mathrm{m}^{2}\right)$ was calculated using NeuriteJ plugin for Image $(\mathrm{NIH})$ software. Results presented as Mean $\pm \mathrm{SD}$; $\mathrm{n}=8$ per condition; ${ }^{* *} \mathrm{p}<0.01 ;{ }^{* * *} \mathrm{p}<0.001$. and MAP-2 (mature neurons) markers. On the other hand no differences were observed in BIII-tubulin levels, another marker of fully mature neurons, as assessed by gene expression (Figs. 1 and 2). Moreover, no differences were observed between the MSC populations, suggesting that the different $\mathrm{CM}$ had the same differentiation potential.

Regarding the effects of MSC CM on axonal growth, the well described DRG-based in vitro model of neurite outgrowth [35] was used. After incubation with MSC CM, we observed that neurite extension from DRGs was promoted by the CM of all populations, in comparison to controls (Fig. 4). Moreover, we verified that ASC $\mathrm{CM}$ induced more neurite extension from the explants, with significant differences regarding BMSC and HUCPVC CM, and control (Fig. 5).

The results herein presented showing the neuroregulatory potential of MSC secretome in neural cultures goes in accordance to some previous studies. For example, our group has shown that the CM of both ASCs and HUCPVCs promoted both proliferation and metabolic activity of hippocampal neurons [26]. We have also observed that BMSC CM improved both neuronal and glial cell survival. In that study different $\mathrm{CM}$ collection times were tested (e.g $24 \mathrm{~h}$ and $96 \mathrm{~h}$ of conditioning). While the former increased the survival of neurons, the later was more prone to improve glial cell survival [36]. High in vitro neuronal differentiation [4,37] and in vivo cell proliferation in the dentate gyrus (DG) of adult rat hippocampus [4] was also found using HUCPVC CM. Interestingly, proteomic analysis on these $\mathrm{CM}$ revealed differences in proteins related with neural cell viability, proliferation and differentiation, namely 14-3-3, Ubiquitin C-Terminal Hydrolase L1 (UCHL1), Heat shock protein (hsp) 70 and Peroxiredoxin-6 (PRDX6), which may explain the above-referred results [36]. Others observed the neurotrophic factors Brain-derived neurotrophic factor (BDNF) and Beta-nerve growth factor ( $\beta$-NGF) to be correlated with the ability of undifferentiated MSCs to induce the survival and neurite outgrowth of neuroblastoma cells and DRGs, respectively [7]. Some other factors not related to MSC so far but that were recently shown to have regenerative and neurotrophic functions are the ASC-secreted Macrophage-colony stimulating factor (MCSF), Matrix metalloproteases (MMPS), Follistatin (FST)-like 1, Mesencephalic astrocyte-derived neurotrophic factor (MANF), and Neuron derived neurotrophic factor (NDNF) [38]. Others like Semaphorins (SEM), Galectins (Gal), Platelet-derived growth factor (PDGF) and Transforming growth factor-beta (TGF- $\beta$ ) were found in the secretome of BMSCs by Cizkova et al. [39]. Altogether, these results suggest the existence of tissue-source based differences, as recently evidenced by Pires et al. [28]. In this proteomic data, the expression of some factors related to neuronal differentiation, namely of PEDF, SEM7A, CDH2 and IL-6 varies in the CM of ASC, BMSCs and HUCPVCs, which would indicate that neurodifferentiation and axonal growth processes mediated by the MSC $\mathrm{CM}$ would be distinct. Still, the role of these factors in the CNS is robustly proved. PEDF, for instance, was shown to induce a neuronal phenotype in cultured human retinoblastoma cells in vitro [40], as well as to contribute to the survival and differentiation of embryonic chick spinal cord motor neurons [41]. $\mathrm{CDH} 2$ was also found to be essential for the neural differentiation of mouse induced pluripotent stem cells [42], and to regulate the pattern of neurodifferentiation in P19 carcinoma cells $[43,44]$. Similarly, IL-6 was recently demonstrated to promote neural differentiation of pluripotent stem cells upon treatment with an immunosuppressive drug [45]. However, the presence of these factors in the CM of MSCs did not translate into a fully maturation into neurons at least using this period of $\mathrm{CM}$ exposure. Nonetheless, we have shown that htNPCs express some of the receptors that have been implicated in the promotion of neurogenesis, 
neurodifferentiation and neuronal migration, namely FLT-1 [46] and TGFBR1 [47], suggesting they might be responsive to the factors present in the MSC CM and that longer culturing periods may potentiate further their differentiation into fully mature neurons (Fig. 3). The putative downstream signaling molecules involved in neuronal differentiation signaling cascades upon activation of these receptors, such as NRP1 [48], SMAD2 [49] and STAT3 $[50,51]$, were not differentially expressed in the cells from the different experimental groups (Fig. 3). In fact, signaling molecules display a very quick turnover, which may have accounted for the lack of significant differences in their expression between groups. Facing these observations, the absence of differences of htNPCs neurodifferentiation herein observed can therefore suggest that the protein levels presented may not be within the optimal dose to fully exert their effects, opening up the possibility to explore new approaches that may promote the secretion of higher doses of these molecules by MSC. Yet, the existence of innumerous distinct protocols among the different studies evaluating the differentiation of neural cultures may also account for different outcomes. Another possible explanation to be considered is the existence of multidirectional function of most of neuroregulatory factors. For example, the upregulation of PEDF in ASC $\mathrm{CM}$ does not necessarily mean it should promote higher levels of neurodifferentiation of htNPCs. On the contrary, this factor is also related to axonal growth [52], which supports the pronounced effects of ASC CM in the levels of neurite outgrowth from DRG explants, depicted in Fig. 5. So, in this case, PEDF appears to be mediating axonal growth over neurodifferentiation. A similar phenomenon might be happening for IL-6. Indeed, this factor was shown to have a role in both neurodifferentiation and axonal growth [53]. But there is a clear upregulation of this factor in HUCPVCs CM in the proteomic analysis [28]. However, the effect of MSC secretome on axonal growth is clearly provided by ASC population (Fig. 5), and not by HUCPVCs, suggesting a poor contribution of IL- 6 in that effect. This is supported by the upregulation levels of SEM7A and Glial-derived nexin (GDN) in the ASC $\mathrm{CM}$, which suggests that it is more likely that these two factors are responsible for mediating axonal growth, rather than IL-6. The potential of SEM7A and GDN in CNS has been in fact shown. The SEM7A-mediated axonal guidance has been proven to be required for proper axon tract formation during embryonic development [54], and to promote spreading and dendricity in human melanocytes [55]. On the other hand, the action of GDN has been also reported several years ago to improve chick sympathetic neurons [56] and hippocampal pyramidal cell [57] neurite extension in vitro. Based on these results, we believe that ASC-mediated neurite outgrowth of DRG explants is mostly based on the combined action of PEDF, SEM7A and GDN. Adding to this, Beta-1,4galactosyltransferase 1 ( $\beta 4$ Gal-T1) protein was found only in the ASC CM [28]. $\beta 4$ Gal-T1 was suggested to regulate the neurite outgrowth on PC12 cells [58] and DRG when co-cultured with Schwann Cells [59]. Moreover, the overexpression of this protein in the lesion site after sciatic nerve crush suggest it involvement in the regeneration of the injured tissue [60]. Therefore, the exclusive presence of this molecule on ASC CM may have indeed improved their impact in the in vitro DRG model of neurite outgrowth herein used. Another multi-functional protein relevant for axonal growth guidance is the extracellular chaperone Clusterin (CLUS), found highly expressed by ASC CM in comparison to BMSC and HUCPVC CM [28]. It is mainly involved in modulating toxic protein deposition in CNS disorders such as Alzheimer's disease [61]. However, studies have also found this protein to contribute to neurite outgrowth of PC12 cells [62] and to induce sensory nerve outgrowth after sciatic nerve transection [63]. Finally, Decorin (DCN), a leucine proteoglycan protein, was also found to be upregulated in ASC CM in our proteomic analysis [28]. This anti-scarring molecule was elsewhere reported to promote robust neurite outgrowth across SCI lesion sites, by reducing the expression of chondroitin sulfate proteoglycan (CPSGs) [64,65].

\section{Conclusions}

This study revealed that the incubation of ASC, BMSC and HUCPVC CM in htNPCs induced their differentiation towards the neuronal phenotype although with no significant differences among them. Moreover, all MSC CM improved neurite/axonal outgrowth in an in vitro model of axonal regeneration based on DRG explants. However, ASC CM provided higher extent of axonal growth when compared to BMSC and HUCPVC. The presence of important neuroregulatory factors in the secretome of MSCs, namely PEDF, CADH2, IL-6, SEM7A and GDN, may explain the observed results. Specifically, the upregulation of PEDF, SEM7A and GDN on ASC CM may be responsible for the higher levels of axonal growth observed with this population. Therefore, these results suggest two mechanisms underlying MSC secretome therapeutic action, namely neurodifferentiation and axonal growth. However, its potential is not limited to these two mechanisms. The modulation of other events such as excitotoxicity, apoptosis, inflammation, should be elucidated to understand their relevance in recovering the normal function of the CNS. The molecular and cellular pathways implicated on them may also be clarified to envisage the future application of MSC secretome in a clinical setting.

\section{Conflicts of interest}

The authors declare no conflict of interests associated with this publication.

\section{Acknowledgments}

The authors acknowledge the financial support by Prémios Santa Casa Neurociências - Prize Melo e Castro for Spinal Cord Injury Research (MC-17-2013 and MC-04-2017); Portuguese Foundation for Science and Technology (Doctoral fellowships PDE/ BDE/113596/2015 and SFRH/BD/120124/2016 to R.C Assunção Silva and B. Mendes-Pinheiro, respectively; Post-doctoral fellowhip to F.G. Teixeira and Patrícia Patrício - SFRH/BPD/118408/2016 and SFRH/BPD/116249/2016; IF Starting Grant to L. Pinto and IF Development Grant to A. J. Salgado); Canada Research Chair in Biomedical Engineering (LAB). This work is funded by national funds through FCT under the scope of grante reference TUBITAK/0007/ 2014. This article has been developed under the scope of the project NORTE-01-0145-FEDER-000013, supported by the Northern Portugal Regional Operational Programme (NORTE 2020), under the Portugal 2020 Partnership Agreement, through the European Regional Development Fund (FEDER). This work has been funded by FEDER funds, through the Competitiveness Factors Operational Programme (COMPETE), and by National funds, through the Foundation for Science and Technology, under the scope of the project POCI-01-0145-FEDER-007038. HUCPVCs and ASCs were kindly provided by Prof. John E. Davies (University of Toronto, Canada) and Prof. Jeff Gimble (LaCell Inc, USA).

\section{References}

[1] A.J. Friedenstein, R.K. Chailakhjan, K.S. Lalykina, The development of fibroblast colonies in monolayer cultures of Guinea-pig bone marrow and spleen cells, Cell Tissue Kinet. 3 (1970) 393-403.

[2] L.R. Zhao, W.M. Duan, M. Reyes, C.D. Keene, C.M. Verfaillie, W.C. Low, Human bone marrow stem cells exhibit neural phenotypes and ameliorate 
neurological deficits after grafting into the ischemic brain of rats, Exp. Neurol. 174 (2002) 11-20.

[3] A. Mahmood, D. Lu, M. Lu, M. Chopp, Treatment of traumatic brain injury in adult rats with intravenous administration of human bone marrow stromal cells, Neurosurgery 53 (2003) 697-702, discussion 702-693.

[4] F.G. Teixeira, M.M. Carvalho, K.M. Panchalingam, A.J. Rodrigues, B. MendesPinheiro, S. Anjo, B. Manadas, L.A. Behie, N. Sousa, A.J. Salgado, Impact of the secretome of human mesenchymal stem cells on brain structure and animal behavior in a rat model of Parkinson's disease, Stem Cell. Transl. Med. 6 (2017) $634-646$.

[5] C.P. Hofstetter, E.J. Schwarz, D. Hess, J. Widenfalk, A. El Manira, D.J. Prockop, L. Olson, Marrow stromal cells form guiding strands in the injured spinal cord and promote recovery, Proc. Natl. Acad. Sci. U.S.A. 99 (2002) 2199-2204.

[6] Y. Akiyama, C. Radtke, J.D. Kocsis, Remyelination of the rat spinal cord by transplantation of identified bone marrow stromal cells, J. Neurosci. : Offic. J. Soc. Neurosci. 22 (2002) 6623-6630.

[7] L. Crigler, R.C. Robey, A. Asawachaicharn, D. Gaupp, D.G. Phinney, Human mesenchymal stem cell subpopulations express a variety of neuro-regulatory molecules and promote neuronal cell survival and neuritogenesis, Exp. Neurol. 198 (2006) 54-64.

[8] A. Jaerve, H.W. Muller, Chemokines in CNS injury and repair, Cell Tissue Res. 349 (2012) 229-248.

[9] N. Alessio, S. Ozcan, K. Tatsumi, A. Murat, G. Peluso, M. Dezawa, U. Galderisi, The secretome of MUSE cells contains factors that may play a role in regulation of stemness, apoptosis and immunomodulation, Cell Cycle 16 (2017) 33-44.

[10] G. Paul, S.V. Anisimov, The secretome of mesenchymal stem cells: potential implications for neuroregeneration, Biochimie 95 (2013) 2246-2256.

[11] Y. Li, J. Chen, X.G. Chen, L. Wang, S.C. Gautam, Y.X. Xu, M. Katakowski, L.J. Zhang, M. Lu, N. Janakiraman, M. Chopp, Human marrow stromal cell therapy for stroke in rat: neurotrophins and functional recovery, Neurology 59 (2002) 514-523.

[12] C. Tran, M.S. Damaser, Stem cells as drug delivery methods: application of stem cell secretome for regeneration, Adv. Drug Deliv. Rev. 82-83 (2015) $1-11$.

[13] X. Wei, L. Zhao, J. Zhong, H. Gu, D. Feng, B.H. Johnstone, K.L. March, M.R. Farlow, Y. Du, Adipose stromal cells-secreted neuroprotective media against neuronal apoptosis, Neurosci. Lett. 462 (2009) 76-79.

[14] G. Lin, G. Wang, L. Banie, H. Ning, A.W. Shindel, T.M. Fandel, T.F. Lue, C.S. Lin, Treatment of stress urinary incontinence with adipose tissue-derived stem cells, Cytotherapy 12 (2010) 88-95.

[15] H.K. Skalnikova, Proteomic techniques for characterisation of mesenchymal stem cell secretome, Biochimie 95 (2013) 2196-2211.

[16] H.O. Kim, S.M. Choi, H.S. Kim, Mesenchymal stem cell-derived secretome and microvesicles as a cell-free therapeutics for neurodegenerative disorders, Tissue Eng. Regen. Med. 10 (2013) 93-101.

[17] J.A. Potian, H. Aviv, N.M. Ponzio, J.S. Harrison, P. Rameshwar, Veto-like activity of mesenchymal stem cells: functional discrimination between cellular responses to alloantigens and recall antigens, J. Immunol. 171 (2003) 3426-3434.

[18] C. Nakanishi, N. Nagaya, S. Ohnishi, K. Yamahara, S. Takabatake, T. Konno, K. Hayashi, M.A. Kawashiri, T. Tsubokawa, M. Yamagishi, Gene and protein expression analysis of mesenchymal stem cells derived from rat adipose tissue and bone marrow, Circ. J. 75 (2011) 2260-2268.

[19] H. Skalnikova, J. Motlik, S.J. Gadher, H. Kovarova, Mapping of the secretome of primary isolates of mammalian cells, stem cells and derived cell lines, Proteomics 11 (2011) 691-708.

[20] A.J. Salgado, R.L. Reis, N.J. Sousa, J.M. Gimble, Adipose tissue derived stem cells secretome: soluble factors and their roles in regenerative medicine, Curr. Stem Cell Res. Ther. 5 (2010) 103-110.

[21] T. Lopatina, N. Kalinina, M. Karagyaur, D. Stambolsky, K. Rubina, A. Revischin, G. Pavlova, Y. Parfyonova, V. Tkachuk, Adipose-derived stem cells stimulate regeneration of peripheral nerves: BDNF secreted by these cells promotes nerve healing and axon growth de novo, PLoS One 6 (2011).

[22] C.S. Lin, H.X. Ning, G.T. Lin, T.F. Lue, Is CD34 truly a negative marker for mesenchymal stromal cells? Cytotherapy 14 (2012) 1159-1163.

[23] R. Vishnubalaji, M. Al-Nbaheen, B. Kadalmani, A. Aldahmash, T. Ramesh, Comparative investigation of the differentiation capability of bone-marrowand adipose-derived mesenchymal stem cells by qualitative and quantitative analysis, Cell Tissue Res. 347 (2012) 419-427.

[24] D. Noel, D. Caton, S. Roche, C. Bony, S. Lehmann, L. Casteilla, C. Jorgensen, B. Cousin, Cell specific differences between human adipose-derived and mesenchymal-stromal cells despite similar differentiation potentials, Exp. Cell Res. 314 (2008) 1575-1584.

[25] S.T.F. Hsiao, A. Asgari, Z. Lokmic, R. Sinclair, G.J. Dusting, S.Y. Lim, R.J. Dilley, Comparative analysis of paracrine factor expression in human adult mesenchymal stem cells derived from bone marrow, adipose, and dermal tissue, Stem Cell. Dev. 21 (2012) 2189-2203.

[26] C.A. Ribeiro, J.S. Fraga, M. Grãos, N.M. Neves, R.L. Reis, J.M. Gimble, N. Sousa, A.J. Salgado, The secretome of stem cells isolated from the adipose tissue and Wharton jelly acts differently on central nervous system derived cell populations, Stem Cell Res. Ther. 3 (2012) 1-7.

[27] J.Y. Hsieh, H.W. Wang, S.J. Chang, K.H. Liao, I.H. Lee, W.S. Lin, C.H. Wu, W.Y. Lin, S.M. Cheng, Mesenchymal stem cells from human umbilical cord express preferentially secreted factors related to neuroprotection, neurogenesis, and angiogenesis, PLoS One 8 (2013).

[28] A.O. Pires, B. Mendes-Pinheiro, F.G. Teixeira, S.I. Anjo, S. Ribeiro-Samy, E.D. Gomes, S.C. Serra, N.A. Silva, B. Manadas, N. Sousa, A.J. Salgado, Unveiling the differences of secretome of human bone marrow mesenchymal stem cells adipose tissue-derived stem cells, and human umbilical cord perivascular cells: a proteomic analysis, Stem Cell. Dev. 25 (2016) 1073-1083.

[29] N.A. Silva, J. Moreira, S. Ribeiro-Samy, E.D. Gomes, R.Y. Tam, M.S. Shoichet, R.L. Reis, N. Sousa, A.J. Salgado, Modulation of bone marrow mesenchyma stem cell secretome by ECM-like hydrogels, Biochimie 95 (2013) 2314-2319.

[30] S.G. Dubois, E.Z. Floyd, S. Zvonic, G. Kilroy, X. Wu, S. Carling, Y.D. Halvorsen, E. Ravussin, J.M. Gimble, Isolation of human adipose-derived stem cells from biopsies and liposuction specimens, Meth. Mol. Biol. 449 (2008) 69-79.

[31] F.G. Teixeira, M.M. Carvalho, A. Neves-Carvalho, K.M. Panchalingam, L.A. Behie, L. Pinto, N. Sousa, A.J. Salgado, Secretome of mesenchymal progenitors from the umbilical cord acts as modulator of neural/glial proliferation and differentiation, Stem Cell Rev. 11 (2015) 288-297.

[32] B.A. Baghbaderani, K. Mukhida, A. Sen, M.S. Kallos, M. Hong, I. Mendez, L.A. Behie, Bioreactor expansion of human neural precursor cells in serumfree media retains neurogenic potential, Biotechnol. Bioeng. 105 (2010) $823-833$.

[33] R.C. Assuncao-Silva, C.C. Oliveira, O. Ziv-Polat, E.D. Gomes, A. Sahar, N. Sousa N.A. Silva, A.J. Salgado, Induction of neurite outgrowth in 3D hydrogel-based environments, Biomed. Mater. 10 (2015), 051001.

[34] E. Oliveira, R.C. Assuncao-Silva, O. Ziv-Polat, E.D. Gomes, F.G. Teixeira, N.A. Silva, A. Shahar, A.J. Salgado, Influence of different ECM-like hydrogels on neurite outgrowth induced by adipose tissue-derived stem cells, Stem Cell. Int. 2017 (2017) 6319129.

[35] I. Allodi, M.S. Guzman-Lenis, J. Hernandez, X. Navarro, E. Udina, In vitro comparison of motor and sensory neuron outgrowth in a 3D collagen matrix, J. Neurosci. Meth. 198 (2011) 53-61.

[36] C.A. Ribeiro, A.J. Salgado, J.S. Fraga, N.A. Silva, R.L. Reis, N. Sousa, The secretome of bone marrow mesenchymal stem cells-conditioned media varies with time and drives a distinct effect on mature neurons and glial cells (primary cultures), J. Tissue Eng. Regen. Med. 5 (2011) 668-672.

[37] J.S. Fraga, N.A. Silva, A.S. Lourenco, V. Goncalves, N.M. Neves, R.L. Reis A.J. Rodrigues, B. Manadas, N. Sousa, A.J. Salgado, Unveiling the effects of the secretome of mesenchymal progenitors from the umbilical cord in different neuronal cell populations, Biochimie 95 (2013) 2297-2303.

[38] N. Kalinina, D. Kharlampieva, M. Loguinova, I. Butenko, O. Pobeguts A. Efimenko, L. Ageeva, G. Sharonov, D. Ischenko, D. Alekseev, O. Grigorieva, V. Sysoeva, K. Rubina, V. Lazarev, V. Govorun, Characterization of secretomes provides evidence for adipose-derived mesenchymal stromal cells subtypes, Stem Cell Res. Ther. 6 (2015).

[39] D. Cizkova, S. Devaux, F. Le Marrec-Croq, J. Franck, L. Slovinska, J. Blasko, J. Rosocha, T. Spakova, C. Lefebvre, I. Fournier, M. Salzet, Modulation properties of factors released by bone marrow stromal cells on activated microglia: an in vitro study, Sci. Rep.-Uk 4 (2014).

[40] F.R. Steele, G.J. Chader, L.V. Johnson, J. Tombrantink, Pigment epitheliumderived factor - neurotrophic activity and identification as a member of the serine protease inhibitor gene family, Proc. Natl. Acad. Sci. U.S.A. 90 (1993) $1526-1530$

[41] L.J. Houenou, A.P. D'Costa, L. Li, V.L. Turgeon, C. Enyadike, E. Alberdi, S.P. Becerra, Pigment epithelium-derived factor promotes the survival and differentiation of developing spinal motor neurons, J. Comp. Neurol. 412 (1999) 506-514.

[42] H.X. Su, L.H. Wang, W.H. Huang, D.J. Qin, J.L. Cai, X.L. Yao, C.Q. Feng, Z.Y. Li Y.T. Wang, K.F. So, G.J. Pan, W.T. Wu, D.Q. Pei, Immediate expression of Cdh2 is essential for efficient neural differentiation of mouse induced pluripotent stem cells, Stem Cell Res. 10 (2013) 338-348.

[43] Y. Wei, T. Harris, G. Childs, Global gene expression patterns during neural differentiation of P19 embryonic carcinoma cells, Differentiation 70 (2002) 204-219.

[44] X. Gao, W. Bian, J. Yang, K. Tang, H. Kitani, T. Atsumi, N. Jing, A role of Ncadherin in neuronal differentiation of embryonic carcinoma P19 cells, Biochem. Biophys. Res. Co 284 (2001) 1098-1103.

[45] A. Ashwini, S.S. Naganur, B. Smitha, P. Sheshadri, J. Prasanna, A. Kumar, Cyclosporine a-mediated IL-6 expression promotes neural induction in pluripotent stem cells, Mol. Neurobiol. 55 (5) (2017) 4267-4279.

[46] T. Hashimoto, X.M. Zhang, B.Y. Chen, X.J. Yang, VEGF activates divergent intracellular signaling components to regulate retinal progenitor cell proliferation and neuronal differentiation, Development 133 (2006) 2201-2210.

[47] T.E. Walshe, L.L. Leach, P.A. D'Amore, TGF-beta signaling is required for maintenance of retinal ganglion cell differentiation and survival, Neuroscience 189 (2011) 123-131.

[48] M. Tillo, L. Erskine, A. Cariboni, A. Fantin, A. Joyce, L. Denti, C. Ruhrberg, VEGF189 binds NRP1 and is sufficient for VEGF/NRP1-dependent neuronal patterning in the developing brain, Development 142 (2015) 314-319.

[49] J. Lu, Y. Wu, N. Sousa, O.F. Almeida, SMAD pathway mediation of BDNF and TGF beta 2 regulation of proliferation and differentiation of hippocampal granule neurons, Development 132 (2005) 3231-3242.

[50] S.J. Park, T. Nakagawa, H. Kitamura, T. Atsumi, H. Kamon, S. Sawa, D. Kamimura, N. Ueda, Y. Iwakura, K. Ishihara, M. Murakami, T. Hirano, IL-6 regulates in vivo dendritic cell differentiation through STAT3 activation, J. Immunol. 173 (2004) 3844-3854.

[51] K. Hawkins, L. Mohamet, S. Ritson, C.L. Merry, C.M. Ward, E-cadherin and, in 
its absence, N-cadherin promotes Nanog expression in mouse embryonic stem cells via STAT3 phosphorylation, Stem Cell. 30 (2012) 1842-1851.

[52] S. Tanimoto, T. Kanamoto, M. Mizukami, H. Aoyama, Y. Kiuchi, Pigment epithelium-derived factor promotes neurite outgrowth of retinal cells, Hiroshima J. Med. Sci. 55 (2006) 109-116.

[53] G. Yang, W.Y. Tang, Resistance of interleukin-6 to the extracellular inhibitory environment promotes axonal regeneration and functional recovery following spinal cord injury, Int. J. Mol. Med. 39 (2017) 437-445.

[54] R.J. Pasterkamp, J.J. Peschon, M.K. Spriggs, A.L. Kolodkin, Semaphorin 7A promotes axon outgrowth through integrins and MAPKs, Nature 424 (2003) 398-405.

[55] G.A. Scott, L.A. McClelland, A.F. Fricke, Semaphorin 7a promotes spreading and dendricity in human melanocytes through beta1-integrins, J. Invest. Dermatol. 128 (2008) $151-161$

[56] A.D. Zurn, H. Nick, D. Monard, A glia-derived nexin promotes neurite outgrowth in cultured chick sympathetic neurons, Dev. Neurosci. 10 (1988) $17-24$.

[57] L. Farmer, J. Sommer, D. Monard, Glia-derived nexin potentiates neurite extension in hippocampal pyramidal cells in vitro, Dev. Neurosci. 12 (1990) 73-80.

[58] Q. Huang, B.D. Shur, P.C. Begovac, Overexpressing cell surface beta 1.4galactosyltransferase in PC12 cells increases neurite outgrowth on laminin, J. Cell Sci. 108 (Pt 2) (1995) 839-847.
[59] A. Shen, J. Yan, F. Ding, X. Gu, D. Zhu, J. Gu, Overexpression of beta-1,4galactosyltransferase I in rat Schwann cells promotes the growth of cocultured dorsal root ganglia, Neurosci. Lett. 342 (2003) 159-162.

[60] M. Yan, C. Cheng, X. Shao, J. Qian, A. Shen, C. Xia, Expression change of beta1,4 galactosyltransferase I, V mRNAs and Galbeta1,4GlcNAc group in rat sciatic nerve after crush, J. Mol. Histol. 39 (2008) 317-328.

[61] J.J. Yerbury, S. Poon, S. Meehan, B. Thompson, J.R. Kumita, C.M. Dobson, M.R. Wilson, The extracellular chaperone clusterin influences amyloid formation and toxicity by interacting with prefibrillar structures, Faseb. J. 21 (2007) 2312-2322.

[62] S.W. Kang, Y.J. Shin, Y.J. Shim, S.Y. Jeong, I.S. Park, B.H. Min, Clusterin interacts with SCLIP (SCG10-like protein) and promotes neurite outgrowth of PC12 cells, Exp. Cell Res. 309 (2005) 305-315.

[63] M.C. Wright, R. Mi, E. Connor, N. Reed, A. Vyas, M. Alspalter, G. Coppola, D.H. Geschwind, T.M. Brushart, A. Hoke, Novel roles for osteopontin and clusterin in peripheral motor and sensory axon regeneration, J. Neurosci. : Offic. J. Soc. Neurosci. 34 (2014) 1689-1700.

[64] J.E. Davies, X.F. Tang, J.C. Bournat, S.J.A. Davies, Decorin promotes plasminogen/plasmin expression within acute spinal cord injuries and by adult microglia in vitro, J. Neurotrauma 23 (2006) 397-408.

[65] K. Minor, X. Tang, G. Kahrilas, S.J. Archibald, J.E. Davies, S.J. Davies, Decorin promotes robust axon growth on inhibitory CSPGs and myelin via a direct effect on neurons, Neurobiol. Dis. 32 (2008) 88-95. 